PROCEEDINGS OF THE

AMERICAN MATHEMATICAL SOCIETY

Volume 131, Number 11, Pages 3395-3405

S 0002-9939(03)06939-9

Article electronically published on March 25, 2003

\title{
THE NUCLEUS FOR RESTRICTED LIE ALGEBRAS
}

\author{
DAVID J. BENSON AND DANIEL K. NAKANO
}

(Communicated by Stephen D. Smith)

\begin{abstract}
The nucleus was a concept first developed in the cohomology theory for finite groups. In this paper the authors investigate the nucleus for restricted Lie algebras. The nucleus is explicitly described for several important classes of Lie algebras.
\end{abstract}

\section{INTRODUCTION}

1.1. The concept of the nucleus was first introduced in $[\mathrm{BCR}]$ in the context of the representation theory and cohomology of a finite group $G$ over a field $k$ of characteristic $p$ dividing $|G|$. The authors were attempting to prove an old conjecture, which states that if $S$ is a simple $k G$-module in the principal block, then $H^{\bullet}(G, S) \neq 0$. This conjecture remains open, but the nucleus has become an important part of the machinery for understanding the relationship between cohomology and representation theory. For example, it is an essential ingredient in the investigation of the thick subcategories of the stable module category [BCRi2, C2]. The nucleus also makes an appearance when we try to understand the annihilator of $\operatorname{Ext}_{k G}{ }^{*}\left(M, M^{\prime}\right)$ in terms of the varieties of $M$ and $M^{\prime}$ [C1].

There are two different versions of the nucleus in the representation theory of finite groups. When the word "nucleus" is used without qualification, it refers to the group theoretical nucleus $Y_{G}$. The other version is called the representation theoretical nucleus $\theta_{G}$. They are both defined as subsets of the cohomology variety $V_{G}$, and one of the main theorems of the subject [Ben2] states that these subsets are equal: $Y_{G}=\theta_{G}$. This theorem indicates further how the group structure is related directly to the representation theory and cohomology. It is easy to see from the definition that the subset $Y_{G}$ is a closed homogeneous subvariety of $V_{G}$. The same is not at all true of $\theta_{G}$, but it may be seen as a consequence of the above theorem.

To be more explicit, the cohomology variety $V_{G}$ is defined to be the maximal ideal spectrum of the cohomology ring $H^{\bullet}(G, k)$ if $p=2$, and of the even degree part of the cohomology ring if $p>2$. The structure of this variety was completely described by Quillen Qu in terms of the set of elementary abelian $p$-subgroups $E$ of $G$ and the conjugations and inclusions between them. If $M$ is a finitely generated

Received by the editors February 20, 2002 and, in revised form, June 20, 2002.

2000 Mathematics Subject Classification. Primary 20G10, 20 G05.

The research of the first author was partially supported by NSF grant DMS-9988110.

The research of the second author was partially supported by NSF grant DMS-0102225. 
$k G$-module, then $V_{G}(M)$ is defined to be the closed homogeneous subvariety of $V_{G}$ determined by the kernel of the map

$$
H^{\bullet}(G, k)=\operatorname{Ext}_{k G}^{\bullet}(k, k) \rightarrow \operatorname{Ext}_{k G}^{\bullet}(M, M)
$$

given by tensoring exact sequences over $k$ with $M$.

The nucleus $Y_{G}$ is defined to be the union of the images of the maps res ${ }_{G, E}^{*}: V_{E} \rightarrow$ $V_{G}$, where $E$ runs over the set of elementary abelian $p$-subgroups of $G$ with the property that the centralizer $C_{G}(E)$ is not $p$-nilpotent. The representation theoretic nucleus $\theta_{G}$ is defined to be the union of the subvarieties $V_{G}(M)$ as $M$ runs over the finitely generated modules in the principal block such that $H^{\bullet}(G, M)=0$ for $\bullet>0$. A description which generalizes to other blocks is that $\theta_{G}$ is equal to the union of the subvarieties $V_{G}(M) \cap V_{G}(N)$ as $M$ and $N$ run over the finitely generated modules in the principal block such that $\operatorname{Ext}_{k G}^{\bullet}(M, N)=0$ for $\bullet>0$ (see [Ben3]).

1.2. Our main goal in this paper is to investigate the corresponding definitions for the $p$-restricted Lie algebras. In this case, the cohomology variety $V_{\mathfrak{g}}$ of $\mathfrak{g}$ was described by Suslin, Friedlander and Bendel [SFB1]. It is homeomorphic to the subvariety of the vector space $\mathfrak{g}$ given by the kernel of the $p$-restriction map $x \mapsto x^{[p]}$. If $G$ is a semisimple algebraic group and $\mathfrak{g}=$ Lie $\mathrm{G}$ with $p>h$, the Coxeter number, then $V_{\mathfrak{g}}$ is the same as the variety $\mathcal{N}$ of nilpotent elements in $\mathfrak{g}$.

We begin by computing the representation theoretic nucleus in the case of a $p$-nilpotent Lie algebra, where the answer is $\{0\}$. This is analogous to the case of a finite $p$-group, where the trivial module is the only simple module. For a Borel subalgebra $\mathfrak{b}$ of $\mathfrak{g}$, the nucleus is the entire cohomology variety. This is somewhat surprising because for finite groups, in odd characteristic the nucleus is always a proper subvariety of the cohomology variety [CR]. This example provides strong indications that there are significant qualitative differences between the group case and the Lie algebra case. Furthermore, in this case there is only one block, and the one-dimensional simple module which induces to the Steinberg module is an example of a simple module with no cohomology. So the original motivating question for finite groups has a negative answer in the case of $p$-restricted Lie algebras. In [CNP Thm 4.3], it was shown that there exists non-projective indecomposable modules in the principal block with no cohomology as long as $\mathfrak{g}$ does not have underlying root system $A_{1}$. Our main theorem, which we prove in Section 3.5, extends this result. We provide an identification of the representation theoretic nucleus for the restricted Lie algebra of a semisimple simply connected algebraic group, under the hypothesis that $p>h+1$.

(1.2.1) Theorem. Let $G$ be a semisimple algebraic group over a field of characteristic $p>h+1$, with $p$-restricted Lie algebra $\mathfrak{g}$. Then the representation theoretic nucleus $\theta_{\mathfrak{g}}$ is equal to the subregular orbit.

The subregular orbit is the (unique) largest closed orbit of $G$ on $\mathcal{N}$ which is not equal to the whole of $\mathcal{N}$. This theorem provides evidence that for an arbitrary restricted Lie algebra $\mathfrak{g}$ there may be a closed subvariety $Y_{\mathfrak{g}}$ related to the structure of the Lie algebra which equals $\theta_{\mathfrak{g}}$. The question of the correct definition for $Y_{\mathfrak{g}}$ in general for $p$-restricted Lie algebras $\mathfrak{g}$ remains open. The proof of the above theorem uses in an essential way the recent calculation of the varieties of Weyl modules given in NPV] as well as the prominent translation functors due to Jantzen. 
(1.2.2) Remark. The paper was initiated at a chance meeting in 1998 at the Plan 9 Music Store in Charlottesville, Virginia.

We end with some questions suggested by our investigations of the representation theoretic nucleus.

\section{Preliminaries}

2.1. Notation. The conventions throughout this paper will closely follow those in [Jan. Let $G$ be a semisimple simply connected algebraic group defined and split over the finite field $\mathbb{F}_{p}$ with $p$ elements. Moreover, let $k$ be the algebraic closure of $\mathbb{F}_{p}$. We will often consider $G$ as an algebraic group scheme over $k$. Let $T$ be a maximal split torus and $\Phi$ be a root system associated to $G$ for a Euclidean space $\mathbb{E}$. The inner product on $\mathbb{E}$ will be denoted by $\langle$,$\rangle and the positive (resp.$ negative) roots will be denoted by $\Phi^{+}$(resp. $\Phi^{-}$). We shall fix a base of simple roots $\Delta$. Let $\alpha^{\vee}=2 \alpha /\langle\alpha, \alpha\rangle$ be the coroot corresponding to $\alpha \in \Phi$ and $\alpha_{0}$ be the highest short root. Set $\rho$ equal to the half sum of positive roots. For any $\alpha \in \Phi$, set $d_{\alpha}=\langle\alpha, \alpha\rangle /\left\langle\alpha_{0}, \alpha_{0}\right\rangle$. The Coxeter number associated to $\Phi$ is $h=\left\langle\rho, \alpha_{0}^{\vee}\right\rangle+1$. Let $W$ be the Weyl group corresponding to $\Phi$ and $W_{p}$ be the affine Weyl group. Let $B$ be a Borel subgroup containing $T$ corresponding to the positive roots and $U$ be the unipotent radical of $B$.

For an affine group scheme $H$ over $k$, let $F: H \rightarrow H^{(1)}$ be the Frobenius morphism and $F^{r}$ be the composition of the Frobenius morphism $r$ times with itself. Set $H_{r}=\operatorname{ker} F^{r}$. For $r=1$, it is well known that there exists a categorical equivalence between $H_{1}$-modules and restricted $\operatorname{Lie}(H)$-modules (or equivalently modules for the restricted enveloping algebra $u(\operatorname{Lie}(H))$. This fact will be used extensively throughout this paper. In particular, we will be interested in the cases when $H=G, B$, and $U$. For $T$ a maximal split torus in $G$, set $G_{r} T=\left(F^{r}\right)^{-1}(T)$ where $F^{r}: G \rightarrow G$.

Let $X(T)$ be the integral weight lattice obtained from $\Phi$. The set $X(T)$ has a partial ordering defined as follows: $\lambda \geq \mu$ if and only if $\lambda-\mu \in \sum_{\alpha \in \Delta} \mathbb{N} \alpha$. The set of dominant integral weights is defined by

$$
X(T)_{+}=\left\{\lambda \in X(T): 0 \leq\left\langle\lambda, \alpha^{\vee}\right\rangle \text { for all } \alpha \in \Delta\right\} .
$$

For $\lambda \in X(T)_{+}$, let $H^{0}(\lambda)=\operatorname{ind}_{B}^{G} \lambda$ and $L(\lambda)=\operatorname{soc}_{G} H^{0}(\lambda)$. This provides a one-to-one correspondence between finite-dimensional simple $G$-modules (labelled by $L(\lambda))$ with $X(T)_{+}$. Furthermore, the set of restricted weights is

$$
X_{r}(T)=\left\{\lambda \in X(T): 0 \leq\left\langle\lambda, \alpha^{\vee}\right\rangle<p^{r} \text { for all } \alpha \in \Delta\right\} .
$$

For $\lambda \in X_{r}(T)$ let $L_{r}(\lambda)=L(\lambda)$. Furthermore, if $\lambda \in X(T)$ we can express $\lambda=\lambda_{0}+p^{r} \lambda^{\prime}$ where $\lambda_{0} \in X_{r}(T)$ and $\lambda^{\prime} \in X(T)$. Set $\widehat{L}_{r}(\lambda)=L\left(\lambda_{0}\right) \otimes p^{r} \lambda^{\prime}$. A complete set of representatives for the isomorphism classes of simple $G_{r}$-modules (resp. $G_{r} T$-modules) is given by $\left\{L_{r}(\lambda): \lambda \in X_{r}(T)\right\}$ (resp. $\left.\left\{\widehat{L}_{r}(\lambda): \lambda \in X(T)\right\}\right)$. The Weyl modules are given by $V(\lambda)=H^{0}\left(-w_{0} \lambda\right)^{*}$ for $\lambda \in X(T)_{+}$. Here $w_{0}$ is the long element in $W$ and ${ }^{*}$ represents taking the dual module.

2.2. Support varieties. Let $A$ be a finite $k$-group and let

$$
H(A, k)= \begin{cases}H^{2 \bullet}(A, k) & \text { if char } k \neq 2 \\ H^{\bullet}(A, k) & \text { if char } k=2\end{cases}
$$


According to [FS], the cohomology ring $R=H(A, k)$ is a commutative, finitely generated $k$-algebra. For finite-dimensional $M, M^{\prime} \in A$-mod, define the relative support variety $V_{A}\left(M, M^{\prime}\right)$ as follows. Yoneda composition defines an action of $R$ on $\operatorname{Ext}_{A}^{\bullet}\left(M, M^{\prime}\right)$. Let $J=J_{A}\left(M, M^{\prime}\right)$ be the annihilator ideal in $H(A, k)$ for this action. Set $V_{K}\left(M, M^{\prime}\right)$ equal to the maximum ideal spectrum of $R / J$. The support variety $V_{A}(M)$ is defined by setting $V_{A}(M)=V_{A}(M, M)$.

Let $A_{0}$ be the block of $A$ containing the trivial module. Set

$$
\begin{aligned}
\mathcal{S} & =\left\{M \in \bmod \left(A_{0}\right): H^{\bullet}(A, M)=0, \bullet>0\right\} \\
& =\left\{M \in \bmod \left(A_{0}\right): V_{A}(k, M)=\{0\}\right\} .
\end{aligned}
$$

The preceding equality follows by the self-injectivity of $A$.

The representation theoretic nucleus of $A, \theta_{A}$, is defined as follows:

$$
\theta_{A}=\bigcup_{M \in \mathcal{S}} V_{A}(M)
$$

From the definition, it is not clear that $\theta_{A}$ is a closed set (i.e., a variety). For finite groups, the representation theoretic nucleus is a variety because one can identify this set with the union $Y_{G}$ of the images of restriction maps from elementary abelian $p$-subgroups whose centralizers are not $p$-nilpotent [Ben2, Cor. 1.3]. In our computations involving infinitesimal Frobenius kernels we will show that the representation theoretic nucleus in these cases is also a variety.

Let $H$ be an algebraic group scheme over $k$ and $\mathfrak{h}=$ Lie $H$. The Lie algebra $\mathfrak{h}$ is a restricted Lie algebra with $p$-mapping $x \rightarrow x^{[p]}$. According to [SFB1 (1.6), (5.11)], $V_{H_{1}}(k)$ is homeomorphic to $\mathcal{N}(\mathfrak{h}):=\left\{x \in \mathfrak{h}: x^{[p]}=0\right\}$. Furthermore, under this identification, if $M$ is an $H_{1}$-module, then $V_{H_{1}}(M)$ is homeomorphic to

$$
\left\{x \in \mathfrak{h}: x^{[p]}=0, \quad M_{\langle x\rangle} \text { is not free }\right\}^{(1)} \cup\{0\} .
$$

We remark that if $M$ is an $H$-module, then $V_{H_{1}}(M)$ is an $H$-stable variety of $\mathfrak{h}$ under conjugation.

2.3. Orbit theory. Let $G$ be a semisimple algebraic group with $\mathfrak{g}=$ Lie $G$. Let $\mathcal{N}$ be the variety of nilpotent elements in $\mathfrak{g}$. For $p>h, \mathcal{N}=\mathcal{N}(\mathfrak{g})$. The group $G$ acts on $\mathcal{N}$ by conjugation and $\mathcal{N}(\mathfrak{g})$ is a $G$-stable subvariety of $\mathcal{N}$.

The orbit theory of $G$ on $\mathcal{N}$ has been well-studied. We refer the reader to [CM], Hum for details. The variety $\mathcal{N}$ has finitely many $G$-orbits. These orbits have been classified. For each $\alpha \in \Phi$, let $x_{\alpha}$ be a root vector. Set $x_{r e g}=\sum_{\alpha \in \Delta} x_{\alpha}$. Then

$$
\mathcal{N}=G \cdot \mathfrak{u}=\overline{G \cdot x_{r e g}}
$$

is called the regular orbit.

Let $I \subseteq \Delta$. Then $\mathfrak{g}=\mathfrak{u}_{I}^{+} \oplus \mathfrak{l}_{I} \oplus \mathfrak{u}_{I}$ has a Levi decomposition. The set $G \cdot \mathfrak{u}_{I}$ can be expressed as the closure of an orbit. Orbits of this form are called Richardson orbits. We should remark that Levi factors which are conjugate under the action of $G$ give rise to the same Richardson orbit. In a specific case one can take $J=\{\alpha\} \subseteq \Delta$. Then $\mathfrak{l}_{J}$ is of type $A_{1}$. The Richardson orbit in this special case is called the subregular orbit. The subregular orbit is the largest proper orbit (i.e. every proper orbit is contained in the subregular orbit). For $I \subseteq \Delta$, let $\Phi_{I}$ be the root system in $\Phi$ generated by $I$. Moreover, for $\lambda \in X(T)$, let

$$
\Phi_{\lambda, p}=\left\{\alpha \in \Phi: d_{\alpha}\left\langle\lambda+\rho, \alpha^{\vee}\right\rangle \in p \mathbb{Z}\right\} .
$$


The following result [NPV, (6.2.1) Thm.] due to Parshall, Vella and the second author involving Richardson orbits will be essential in our computations of the representation theoretic nucleus.

(2.3.1) Theorem. For $\lambda \in X(T)_{+}$and $p$ good, choose $I \subseteq \Delta$ so that $w\left(\Phi_{\lambda, p}\right)=\Phi_{I}$ for some $w \in W$. Then $V_{G_{1}}\left(H^{0}(\lambda)\right)=G \cdot \mathfrak{u}_{I}$.

2.4. Translation functors. Let $\bar{C}_{\mathbb{Z}}=\left\{\lambda \in X(T): 0 \leq\left\langle\lambda+\rho, \alpha^{\vee}\right\rangle \leq p\right.$ for all $\left.\alpha \in \Phi^{+}\right\}$. Moreover, let $M$ be a $G$-module and let $\operatorname{pr}_{\sigma}(M)$ be the sum of all submodules of $M$ with $G$-composition factors with high weight in $W_{p} \cdot \sigma$. If $Z$ is a set of $W_{p}$-orbits in $X(T)$ (under the dot action), then

$$
M \cong \bigoplus_{\mu \in Z} \operatorname{pr}_{\mu}(M)
$$

We will use the translation functors Jan II 7.6] first constructed by Jantzen. If $\lambda, \mu \in \bar{C}_{\mathbb{Z}}$, let $\nu_{1} \in W(\mu-\lambda) \cap X(T)_{+}$. For any $G$-module $M$, let

$$
T_{\lambda}^{\mu}(M)=\operatorname{pr}_{\mu}\left(\operatorname{pr}_{\lambda}(M) \otimes H^{0}\left(\nu_{1}\right)\right) .
$$

The functor $T_{\lambda}^{\mu}$ is exact. Moreover, $T_{\lambda}^{\mu}$ and $T_{\mu}^{\lambda}$ are adjoint to each other. The functor $T_{\lambda}^{\mu}$ commutes with the forgetful functor from $G$-modules to $G_{r} T$-modules. Consequently, $T_{\lambda}^{\mu}$ and $T_{\mu}^{\lambda}$ are adjoint in the category of $G_{r} T$-modules. Many of the results for $G$-modules (in [Jan, II Ch. 7]) have analogous statements in the category of $G_{r} T$-modules (see [Jan, II 9.19]). We will rely on these results in our computations. Finally observe that since $V_{G_{1}}\left(M_{1} \otimes M_{2}\right)=V_{G_{1}}\left(M_{1}\right) \cap V_{G_{1}}\left(M_{2}\right)$ and $V_{G_{1}}\left(M_{1} \oplus M_{2}\right)=V_{G_{1}}\left(M_{1}\right) \cup V_{G_{1}}\left(M_{2}\right)$, we have

$$
V_{G_{1}}\left(T_{\lambda}^{\mu}(M)\right) \subseteq V_{G_{1}}(M) \cap V_{G_{1}}\left(H^{0}\left(\nu_{1}\right)\right) \subseteq V_{G_{1}}(M)
$$

\section{Computation of the Representation theoretic nucleus}

3.1. Carlson and Robinson [CR, Thm. 4.1] have demonstrated that for group algebras of a finite group, the nucleus must be a proper subvariety of the support variety of the trivial module if the underlying field does not have characteristic two. For fields of characteristic two there are examples where the nucleus is not proper CR, Prop. 5.2]. In our first result we show that for the Borel subalgebra of a classical Lie algebra the representation theoretic nucleus need not be proper. This result is independent of the characteristic of the field.

(3.1.1) Proposition. Let $G$ be a semisimple algebraic group. Then

(a) $\theta_{U_{1}}=\{0\}$;

(b) $\theta_{B_{1}}=\mathcal{N}(\mathfrak{u})$.

In particular if $p>h$, then $\theta_{B_{1}}=\mathfrak{u}$.

Proof. (a) Since $\mathfrak{u}$ is a $p$-nilpotent Lie algebra, the only simple $U_{1}$-module is the trivial module $k$. If $H^{\bullet}\left(U_{1}, M\right)=0$ for $\bullet>0$, then $M$ is a projective $U_{1}$-module; thus $\theta_{U_{1}}=\{0\}$.

(b) Let $(p-1) \rho \in X(T)+$ be the dominant weight corresponding to the Steinberg module. We have

$$
L((p-1) \rho)=\operatorname{ind}_{B_{1}}^{G_{1}}(p-1) \rho .
$$

Since $L((p-1) \rho)$ is a projective $G_{1}$-module, it follows that

$$
H^{\bullet}\left(B_{1},(p-1) \rho\right)=H^{\bullet}\left(G_{1}, L((p-1) \rho)\right)=0
$$


for $\bullet>0$. The group scheme $B_{1}$ has one block. Moreover, $(p-1) \rho$ is a onedimensional $B_{1}$-module so $V_{B_{1}}((p-1) \rho)=\mathcal{N}(\mathfrak{b})=\mathcal{N}(\mathfrak{u})$. Consequently, $\theta_{B_{1}}=$ $\mathcal{N}(\mathfrak{u})$.

3.2. Let $\uparrow$ be the Strong Linkage relation on $X(T)$ as defined in Jan II 6.4]: $\lambda \uparrow \mu$ if and only if there exists $\mu_{1}, \mu_{2}, \ldots, \mu_{t} \in X(T)$ and reflections $s_{1}, s_{2}, \ldots, s_{t+1} \in W_{p}$ such that

$$
\lambda \leq s_{1} \cdot \lambda=\mu_{1} \leq s_{2} \cdot \mu_{1}=\mu_{2} \leq \cdots \leq s_{t} \cdot \mu_{t-1}=\mu_{t} \leq s_{t+1} \cdot \mu_{t}=\mu .
$$

Here $\cdot$ denotes the "dot action" of the affine Weyl group.

Let $\mathcal{B}_{\lambda}\left(G_{1}\right)$ (resp. $\left.\mathcal{B}_{\lambda}\left(G_{1} T\right)\right)$ be the block for $G_{1}$ (resp. $G_{1} T$ ) that contains $L_{1}(\lambda)$ (resp. $\left.\widehat{L}_{1}(\lambda)\right)$. For brevity we will write $\mu \in \mathcal{B}_{\lambda}\left(G_{1}\right)$ to indicate that the simple $G_{1}$-module $L_{1}(\mu)$ is in the block $\mathcal{B}_{\lambda}\left(G_{1}\right)$. A similar statement can be made for $\lambda \in \mathcal{B}_{0}\left(G_{1} T\right)$. The principal block for $G_{1}\left(\operatorname{resp} . G_{1} T\right)$ is $\mathcal{B}_{0}\left(G_{1}\right)\left(\operatorname{resp} . \mathcal{B}_{0}\left(G_{1} T\right)\right)$. From [Jan, II $9.19(1)], \lambda \in \mathcal{B}_{0}\left(G_{1} T\right)$ if and only if $\lambda \in W \cdot 0+p X(T)$. Moreover, $\lambda \in \mathcal{B}_{0}\left(G_{1}\right)\left(\lambda \in X_{1}(T)\right)$ if and only if $\lambda \equiv W \cdot 0(\bmod p X(T))$.

(3.2.1) Proposition. If $\lambda \in \mathcal{B}_{0}\left(G_{1}\right)\left(\lambda \in X_{1}(T)\right)$, then there exists $\nu \in X(T)$ such that

(i) $0 \uparrow \lambda+p \nu$;

(ii) $\lambda+p \nu \in X(T)_{+}$with $\nu \in X(T)_{+}$.

Proof. Since $\lambda \in \mathcal{B}_{0}\left(G_{1}\right)$, there exists $w \in W, \zeta \in X(T)$ such that $w \cdot \lambda+p \zeta=0$, so $w \cdot \lambda=-p \zeta$. From [Jan, II 6.4(5)], we have $w \cdot \lambda \uparrow \lambda$ for all $w \in W$. This implies that $-p \zeta \uparrow \lambda$ or $0 \uparrow \lambda+p \zeta$. Let $\beta$ be the highest (long) root. By applying Jan, II 6.4(3)(4)], $\lambda+p \zeta \uparrow \lambda+p \zeta+p s \beta$ for $s \in \mathbb{Z}^{+}$. Therefore, $0 \uparrow \lambda+p \zeta+p s \beta$ for $s \in \mathbb{Z}^{+}$. For $s$ sufficiently large $\zeta+s \beta \in X(T)_{+}$with $\lambda+p(\zeta+s \beta) \in X(T)_{+}$ because $\left\langle\beta, \alpha^{\vee}\right\rangle>0$ for all $\alpha \in \Delta$.

3.3. The following theorem shows that for classical Lie algebras for $p \geq h$, the representation theoretic nucleus is contained in the subregular nilpotent orbit, and is thus a proper subvariety of the support variety of the trivial module.

(3.3.1) Theorem. Let $G$ be a semisimple algebraic group with $p \geq h$. Then $\theta_{G_{1}} \subseteq G \cdot \mathfrak{u}_{J}$ where $J=\{\alpha\} \subseteq \Delta$.

Proof. Let $\mathcal{D}=\left\{\sigma \in X(T)_{+}: 0 \uparrow \sigma\right\}$ and $\mathcal{S}=\left\{M \in \bmod \left(\mathcal{B}_{0}\left(G_{1}\right)\right): H^{\bullet}\left(G_{1}, M\right)=\right.$ $0, \bullet>0\}$. We will first show that $V_{G_{1}}\left(H^{0}(\sigma), M\right) \subseteq G \cdot \mathfrak{u}_{J}$ for all $\sigma \in \mathcal{D}$ and $M \in \mathcal{S}$. This will be accomplished by using induction on the ordering in $\mathcal{D}$. First observe that $\operatorname{Ext}_{G_{1}}^{\bullet}\left(H^{0}(0), M\right)=\operatorname{Ext}_{G_{1}}^{\bullet}(k, M)=0$ for $\bullet>0$; thus $V_{G_{1}}\left(H^{0}(0), M\right)=\{0\} \subseteq$ $G \cdot \mathfrak{u}_{J}$.

Now let $\sigma \in \mathcal{D}, \sigma \neq 0$ and assume that for all $\eta \in \mathcal{D}$ with $0 \uparrow \eta$ and $\eta<\sigma$, $V_{G_{1}}\left(H^{0}(\eta), M\right) \subseteq G \cdot \mathfrak{u}_{J}$. Since $\sigma \neq 0$ and $0 \uparrow \sigma$, there exists a reflection $s$ in $W_{p}$ such that $s \cdot \sigma<\sigma$ and $0 \uparrow s \cdot \sigma$. Let $\mu^{\prime}$ be such that $\operatorname{Stab}_{W_{p}} \mu^{\prime}=\{1, s\}$. There exists a $w \in W_{p}$ such that for some $\mu \in \bar{C}_{\mathbb{Z}}, w \cdot 0=\sigma$ and $w \cdot \mu=\mu^{\prime}$. According to [Jan, II 7.13 Prop.], the module $T_{\mu}^{\lambda}\left(H^{0}\left(\mu^{\prime}\right)\right)=T_{\mu}^{\lambda}\left(H^{0}(w \cdot \mu)\right)$ has composition factors with multiplicity at most one of the form $H^{0}(\sigma)$ and $H^{0}(s \cdot \sigma)$.

Without loss of generality we may assume that there exists an exact sequence of the form

$$
0 \rightarrow H^{0}(s \cdot \sigma) \rightarrow T_{\mu}^{\lambda}\left(H^{0}\left(\mu^{\prime}\right)\right) \rightarrow H^{0}(\sigma) \rightarrow 0
$$


We remark that the order of the composition factors may be reversed, but the argument is the same in either case. Also, note that it is possible to have $s \cdot \sigma \notin$ $X(T)_{+}$in which case $H^{0}(s \cdot \sigma)=0$. The short exact sequence above induces a long exact sequence in cohomology:

$\left.\cdots \rightarrow \operatorname{Ext}_{G_{1}}^{i}\left(H^{0}(\sigma), M\right) \rightarrow \operatorname{Ext}_{G_{1}}^{i}\left(T_{\mu}^{\lambda}\left(H^{0}\left(\mu^{\prime}\right)\right), M\right) \rightarrow \operatorname{Ext}_{G_{1}}^{i}\left(H^{0}(s \cdot \sigma)\right), M\right) \rightarrow \cdots$.

This implies that

$$
\begin{aligned}
V_{G_{1}}\left(H^{0}(\sigma), M\right) & \subseteq V_{G_{1}}\left(H^{0}(s \cdot \sigma), M\right) \cup V_{G_{1}}\left(T_{\mu}^{\lambda}\left(H^{0}\left(\mu^{\prime}\right)\right), M\right) \\
& \subseteq G \cdot \mathfrak{u}_{J} \cup V_{G_{1}}\left(T_{\mu}^{\lambda}\left(H^{0}\left(\mu^{\prime}\right)\right), M\right) .
\end{aligned}
$$

By using the definition of the translation functor and Theorem 2.3.1 with $\Phi_{\mu^{\prime}, p}$ of type $A_{1}$, we have

$$
\begin{aligned}
V_{G_{1}}\left(T_{\mu}^{\lambda}\left(H^{0}\left(\mu^{\prime}\right)\right), M\right) & \subseteq V_{G_{1}}\left(T_{\mu}^{\lambda}\left(H^{0}\left(\mu^{\prime}\right)\right)\right) \\
& \subseteq V_{G_{1}}\left(H^{0}\left(\mu^{\prime}\right)\right) \\
& \subseteq G \cdot \mathfrak{u}_{J} .
\end{aligned}
$$

Hence, $V_{G_{1}}\left(H^{0}(\sigma), M\right) \subseteq G \cdot \mathfrak{u}_{J}$.

Next we will show that $V_{G_{1}}(L(\lambda), M) \subseteq G \cdot \mathfrak{u}_{J}$ for all $\lambda \in \mathcal{D}$ and $M \in \mathcal{S}$. Again we will use induction on the ordering of weights in $\mathcal{D}$. For $M \in \mathcal{S}$, one has

$$
V_{G_{1}}(k, M)=V_{G_{1}}(L(0), M)=\{0\} \subseteq G \cdot \mathfrak{u}_{J} .
$$

If $\lambda \in \mathcal{D}$, there exists a short exact sequence of $G$-modules of the form

$$
0 \rightarrow L(w \cdot 0) \rightarrow H^{0}(w \cdot 0) \rightarrow N \rightarrow 0
$$

with all composition factors in $N$ of the form $L(\sigma)$ with $\sigma \uparrow \lambda$ and $\sigma<\lambda$. By the same argument as in the preceding paragraph (using the long exact sequence), we have

$$
\begin{aligned}
V_{G_{1}}(L(w \cdot 0), M) & \subseteq V_{G_{1}}\left(H^{0}(w \cdot 0), M\right) \cup V_{G_{1}}(N, M) \\
& \subseteq G \cdot \mathfrak{u}_{J} \cup G \cdot \mathfrak{u}_{J} \\
& \subseteq G \cdot \mathfrak{u}_{J} .
\end{aligned}
$$

The second-to-last line follows by using the induction hypothesis and the assertion proved in the preceding paragraph.

Finally, let $\lambda \in X_{1}(T)$ and $L_{1}(\lambda)$ be a simple $G_{1}$-module in $\mathcal{B}_{0}\left(G_{1}\right)$. We claim that $V_{G_{1}}\left(L_{1}(\lambda), M\right) \subseteq G \cdot \mathfrak{u}_{J}$ for all $M \in \mathcal{S}$. Since $\lambda \in \mathcal{B}_{0}\left(G_{1}\right)$, by Proposition 3.2.1, there exists $\nu \in X(T)_{+}$such that $0 \uparrow \lambda+p \nu$ and $\lambda+p \nu \in X(T)_{+}$. Therefore,

$$
L(\lambda+p \nu) \cong L(\lambda) \otimes L(\nu)^{(1)} \cong L_{1}(\lambda) \otimes L(\nu)^{(1)} .
$$

This implies that $\operatorname{Ext}_{G_{1}}^{\bullet}(L(\lambda+p \nu), M) \cong \operatorname{Ext}_{G_{1}}^{\bullet}\left(L_{1}(\lambda), M\right) \otimes L(\nu)^{(1)}$. Therefore, from the claim in the preceding paragraph,

$$
V_{G_{1}}\left(L_{1}(\lambda), M\right)=V_{G_{1}}(L(\lambda+p \nu), M) \subseteq G \cdot \mathfrak{u}_{J}
$$

for all $M \in \mathcal{S}$. Now suppose that $M \in \mathcal{S}$. Then

$$
V_{G_{1}}(M)=V_{G}\left(\bigoplus_{\lambda \in \mathcal{B}_{0}\left(G_{1}\right)} L_{1}(\lambda), M\right) \subseteq G \cdot \mathfrak{u}_{J} .
$$

Consequently, $\theta_{G_{1}} \subseteq G \cdot \mathfrak{u}_{J}$. 
3.4. In this section we will investigate the properties of support varieties of induced modules relative to the translation functor.

(3.4.1) Proposition. Let $G$ be a semisimple algebraic group with $p$ good. Let $\lambda, \mu \in \bar{C}_{\mathbb{Z}}$ such that $\mu$ belongs to the closure of the facet containing $\lambda$. If $w \cdot \mu \in$ $X(T)_{+}$where $w \in W_{p}$, then $V_{G_{1}}\left(T_{\mu}^{\lambda}\left(H^{0}(w \cdot \mu)\right)\right)=V_{G_{1}}\left(H^{0}(w \cdot \mu)\right)$.

Proof. Set $\mu^{\prime}=w \cdot \mu$. Since $T_{\mu}^{\lambda}\left(H^{0}\left(\mu^{\prime}\right)\right)$ is a $G_{1} T$-module, it follows by Jan, I 6.9 (4)(5)] for $\sigma \in X_{1}(T)$,

$$
\begin{aligned}
\operatorname{Ext}_{G_{1}}^{\bullet}\left(L_{1}(\sigma), T_{\mu}^{\lambda}\left(H^{0}\left(\mu^{\prime}\right)\right)\right) & \cong \bigoplus_{\nu \in X(T)} \operatorname{Ext}_{G_{1} T}^{\bullet}\left(\widehat{L}_{1}(\sigma+p \nu), T_{\mu}^{\lambda}\left(H^{0}\left(\mu^{\prime}\right)\right)\right) \\
& \cong \bigoplus_{\nu \in X(T)} \operatorname{Ext}_{G_{1} T}^{\bullet}\left(T_{\lambda}^{\mu}\left(\widehat{L}_{1}(\sigma+p \nu)\right), H^{0}\left(\mu^{\prime}\right)\right) .
\end{aligned}
$$

Therefore,

$$
\begin{aligned}
\operatorname{Ext}_{G_{1}}^{\bullet}\left(\bigoplus_{\sigma \in \mathcal{B}_{\lambda}\left(G_{1}\right)} L(\sigma), T_{\mu}^{\lambda}\left(H^{0}\left(\mu^{\prime}\right)\right)\right) \\
\quad \cong \bigoplus_{\sigma \in \mathcal{B}_{\lambda}\left(G_{1}\right)} \bigoplus_{\nu \in X(T)} \operatorname{Ext}_{G_{1} T}^{\bullet}\left(T_{\lambda}^{\mu}\left(\widehat{L}_{1}(\sigma+p \nu)\right), H^{0}\left(\mu^{\prime}\right)\right) .
\end{aligned}
$$

Let $\gamma \in X(T)$ be such that $\widehat{L}_{1}(\gamma) \in \widehat{\mathcal{B}}_{\mu}\left(G_{1} T\right)$. The proof of [Jan, II 7.15] can be adapted for $G_{1} T$-modules to show that there exists $\widehat{L}_{1}(\eta)$ with $\widehat{L}_{1}(\eta) \in \widehat{B}_{\lambda}\left(G_{1} T\right)$ such that

$$
T_{\lambda}^{\mu}\left(\widehat{L}_{1}(\eta)\right)=\widehat{L}_{1}(\gamma)
$$

Hence, by using (3.4.2),

$$
\begin{aligned}
\operatorname{Ext}_{G_{1}}^{\bullet}\left(\bigoplus_{\sigma \in \mathcal{B}_{\lambda}\left(G_{1}\right)} L(\sigma), T_{\mu}^{\lambda}\left(H^{0}\left(\mu^{\prime}\right)\right)\right) & \cong \bigoplus_{\eta \in \mathcal{B}_{\lambda}\left(G_{1} T\right)} \operatorname{Ext}_{G_{1} T}^{\bullet}\left(T_{\lambda}^{\mu}\left(\widehat{L}_{1}(\eta)\right), H^{0}\left(\mu^{\prime}\right)\right) \\
& \cong \operatorname{Ext}_{G_{1} T}^{\bullet}\left(\bigoplus_{\eta \in \mathcal{B}_{\lambda}\left(G_{1} T\right)} T_{\lambda}^{\mu}\left(\widehat{L}_{1}(\eta)\right), H^{0}\left(\mu^{\prime}\right)\right) \\
& \supseteq \operatorname{Ext}_{G_{1} T}^{\bullet}\left(\bigoplus_{\gamma \in \mathcal{B}_{\mu}\left(G_{1} T\right)} \widehat{L}_{1}(\gamma), H^{0}\left(\mu^{\prime}\right)\right) \\
& \cong \operatorname{Ext}_{G_{1}}^{\bullet}\left(\bigoplus_{\zeta \in \mathcal{B}_{\mu}\left(G_{1}\right)} L_{1}(\zeta), H^{0}\left(\mu^{\prime}\right)\right) .
\end{aligned}
$$

Therefore, $\operatorname{dim} V_{G_{1}}\left(H^{0}\left(\mu^{\prime}\right)\right) \leq \operatorname{dim} V_{G_{1}}\left(T_{\mu}^{\lambda}\left(H^{0}\left(\mu^{\prime}\right)\right)\right)$ from the inclusion above and NPV (2.2.2) Thm.]. In Section 2.4 we have seen that

$$
V_{G_{1}}\left(T_{\mu}^{\lambda}\left(H^{0}\left(\mu^{\prime}\right)\right)\right) \subseteq V_{G_{1}}\left(H^{0}\left(\mu^{\prime}\right)\right) .
$$

Since $V_{G_{1}}\left(H^{0}\left(\mu^{\prime}\right)\right)$ is irreducible [NPV, (6.3.1) Cor.], it follows that

$$
V_{G_{1}}\left(T_{\mu}^{\lambda}\left(H^{0}\left(\mu^{\prime}\right)\right)\right)=V_{G_{1}}\left(H^{0}\left(\mu^{\prime}\right)\right) .
$$

3.5. An open question in [CNP, (5.1)] was to determine when there exist indecomposable $G$-modules $M$ in the principal block for $G_{r}$ (not projective as $G_{r}$-modules) such that $H^{\bullet}\left(G_{r}, M\right)=0$. An affirmative answer was given for $r \geq 2$ in [BN] (4.1.1) Thm.]. The following theorem provides an affimative answer for $r=1$ as long as $G$ does not have underlying root system $A_{1}$. 
(3.5.1) Theorem. Let $G$ be a semisimple algebraic group with $p>h+1$. There exists a $G$-module $M$ such that

(a) $M$ is in the principal block of $G_{1}$;

(b) $H^{\bullet}\left(G_{1}, M\right)=0$ for $\bullet>0$;

(c) $V_{G_{1}}(M)=G \cdot \mathfrak{u}_{J}$ where $J=\{\alpha\} \subseteq \Delta$.

Proof. Let $\mu^{\prime}=w \cdot \mu \in X(T)_{+}$where $w \in W_{p}$ and $\mu \in \bar{C}_{\mathbb{Z}}$. Also assume that $\Phi_{\mu, p}$ is of type $A_{1}$. Consider $M=T_{\mu}^{0}\left(H^{0}\left(\mu^{\prime}\right)\right)$. By construction $M$ is in $\mathcal{B}_{0}\left(G_{1}\right)$. Moreover, by Proposition 3.4.1 (with $\lambda=0$ ) and Theorem 2.3.1, $V_{G_{1}}(M)=G \cdot \mathfrak{u}_{J}$ where $J=\{\alpha\} \subseteq \Delta$. We need to show that $H^{\bullet}\left(G_{1}, M\right)=0$ for $\bullet>0$. Now

$$
\begin{aligned}
H^{\bullet}\left(G_{1}, M\right) & \cong \operatorname{Ext}_{G_{1}}^{\bullet}\left(k, T_{\mu}^{0}\left(H^{0}\left(\mu^{\prime}\right)\right)\right) \\
& \subseteq \operatorname{Ext}_{G_{1}}^{\bullet}\left(k, H^{0}\left(\mu^{\prime}\right) \otimes H^{0}(w(-\mu))\right) \\
& \cong \operatorname{Ext}_{G_{1}}^{\bullet}\left(H^{0}(w(-\mu))^{*}, H^{0}\left(\mu^{\prime}\right)\right) \\
& \cong \operatorname{Ext}_{G_{1}}^{\bullet}\left(V\left(w_{0} w \mu\right), H^{0}\left(\mu^{\prime}\right)\right) .
\end{aligned}
$$

Let $L(\lambda)$ be a $G$-composition factor of $H^{0}(\sigma)$. Then $\lambda \uparrow \sigma$ and $\lambda \in W \cdot \sigma+p X(T)$. Decompose $\lambda=\lambda_{0}+p \nu$ and $\sigma=\sigma_{0}+p \nu^{\prime}$ where $\lambda_{0}, \sigma_{0} \in X_{1}(T)$ and $\nu, \nu^{\prime} \in X(T)_{+}$. Observe that $L(\lambda)=L_{1}\left(\lambda_{0}\right) \otimes L(\nu)^{(1)}$. Therefore, if $L_{1}\left(\lambda_{0}\right)$ is a $G_{1}$-composition factor of $H^{0}(\lambda)$, then $\lambda_{0} \in W \cdot \sigma+p X(T)$. We can conclude that if $H^{\bullet}\left(G_{1}, M\right)=0$, then $\operatorname{Ext}_{G_{1}}^{\bullet}\left(V\left(w_{0} w \mu\right), H^{0}\left(\mu^{\prime}\right)\right) \neq 0$ for some $\bullet \geq 0$ which implies that $w_{0} w \mu \in$ $W \cdot \mu+p X(T)$.

We can find a fundamental weight $\omega_{\alpha}$ for some $\alpha \in \Delta$ such that $-2 \leq\left\langle\omega_{\alpha}, \gamma^{\vee}\right\rangle \leq$ 2 for all $\gamma \in \Phi$. Let $\mu=-\sum_{\beta \in \Delta-\{\alpha\}} \omega_{\beta}$. Then $\mu \in \bar{C}_{\mathbb{Z}}$. Furthermore, $\Phi_{\mu, p}$ is of type $A_{1}$.

Now choose $\mu^{\prime}=w \cdot \mu \in X(T)_{+}$where $w \in W_{p}$ such that $w_{0} w \neq \mathrm{id}+p \sigma$ for some $\sigma \in X(T)$. From our analysis above we can actually say that $w_{0} w \mu \in W \cdot \mu+p X(T)$ for $w \in W$ with $w_{0} w \neq$ id. Express $w_{0} w \mu=w_{1} \cdot \mu+p \nu_{1}$ for some $w_{1} \in W$ and $\nu_{1} \in X(T)$. Let $\gamma \in \Delta$. Then

$$
\begin{aligned}
\left|p\left\langle\nu_{1}, \gamma^{\vee}\right\rangle\right| & =\left|\left\langle w_{0} w \mu-w_{1} \cdot \mu, \gamma^{\vee}\right\rangle\right| \\
& =\left|\left\langle w_{0} w \mu-w_{1}(\mu+\rho)+\rho, \gamma^{\vee}\right\rangle\right| \\
& =\left|\left\langle\mu,\left(w_{0} w\right)^{-1} \gamma^{\vee}\right\rangle-\left\langle\omega_{\alpha}, w_{1}^{-1} \gamma^{\vee}\right\rangle+1\right| \\
& \leq h+1 .
\end{aligned}
$$

It follows that for $p>h+1, \nu_{1}=0$; thus $w_{0} w \mu \in W \cdot \mu$. One can now apply [Jan, II (7.7b) Lemma] to conclude that $w_{0} w=\mathrm{id}$. This is a contradiction. Consequently, $w_{0} w \mu \notin W \cdot \mu+p X(T)$ and $H^{\bullet}\left(G_{1}, T_{\mu}^{0}\left(H^{0}\left(\mu^{\prime}\right)\right)\right)=0$.

We can now show that the representation theoretic nucleus for $G_{1}$ for $p>h+1$ is precisely the subregular orbit.

(3.5.2) Corollary. Let $G$ be a semisimple algebraic group with $p>h+1$. Then $\theta_{G_{1}}=G \cdot \mathfrak{u}_{J}$ where $J=\{\alpha\} \subseteq \Delta$.

Proof. From Theorem 3.3.1, we have $\theta_{G_{1}} \subseteq G \cdot \mathfrak{u}_{J}$. On the other hand, by Theorem 3.5.1, there exists an $M \in \mathcal{S}$ with $V_{G_{1}}(M)=G \cdot \mathfrak{u}_{J}$, thus $\theta_{G_{1}}=G \cdot \mathfrak{u}_{J}$.

In view of the equivalence of categories between the $G_{1}$-modules and the $u(\mathfrak{g})$ modules, Theorem 1.2.1 follows immediately from the above corollary. 


\section{FURThER DIRECTIONS}

4.1. In this section, we describe some questions opened up by this work. Let $\mathfrak{g}$ be a $p$-restricted Lie algebra.

(4.1.1) Question. What is $\theta_{\mathfrak{g}}$ in the case of the $p$-restricted Lie algebra of a semisimple algebraic group with $p \leq h+1$ ?

(4.1.2) Question. What is $\theta_{\mathfrak{g}}$ for $\mathfrak{g}$ a restricted Lie algebra of Cartan type?

(4.1.3) Question. What is the correct definition of the nucleus $Y_{\mathfrak{g}}$ in general? It should be defined just in terms of the Lie structure, without mentioning cohomology. There should then be a theorem stating that it is equal to the representation theoretic nucleus $\theta_{\mathfrak{g}}$.

(4.1.4) Question. Is it true that if $M$ and $M^{\prime}$ are finitely generated modules in the principal block of $u(\mathfrak{g})$, then $V_{\mathfrak{g}}(M) \cap V_{\mathfrak{g}}\left(M^{\prime}\right) \subseteq V_{\mathfrak{g}}\left(M, M^{\prime}\right) \cup \theta_{\mathfrak{g}}$ ? This was proved in the finite group case in [C1], but the proof does not obviously translate.

(4.1.5) Question. Describe the thick subcategories of the stable category of finitely generated modules in the principal block of $\mathfrak{g}$. If the situation is analogous to the finite group case [BCRi2], then modulo modules whose variety is contained in the nucleus, one should be able to answer the question in terms of varieties. Each line through the origin in the nucleus should then support an infinite number of thick subcategories.

\section{REFERENCES}

[BN] C. P. Bendel and D. K. Nakano, Complexes and vanishing of cohomology for group schemes, J. Algebra 214 (1999), 668-713. MR 2001a:16015

[Ben1] D. J. Benson, Representations and cohomology, II, Cambridge Univ. Press, 1991. MR 93g:20099

[Ben2] D. J. Benson, Cohomology of modules in the principal block of a finite group, New York J. Math. 1 (1995), 196-205. MR 96h:20095

[Ben3] D. J. Benson, The nucleus, and extensions between modules for a finite group, Representations of Algebras, Proceedings of the Ninth International Conference (Beijing 2000), Beijing Normal University Press, 2002.

[BCRi1] D. J. Benson, J. F. Carlson and J. Rickard, Complexity and varieties for infinitely generated modules, II, Math. Proc. Camb. Phil. Soc. 120 (1996), 597-615. MR 97f:20008

[BCRi2] D. J. Benson, J. F. Carlson and J. Rickard, Thick subcategories of the stable module category, Fundamenta Mathematicae 153 (1997), 59-80. MR 98g:20021

[BCR] D. J. Benson, J. F. Carlson and G. R. Robinson, On the vanishing of group cohomology, J. Algebra 131 (1990), 40-73. MR 91c:20073

[C1] J. F. Carlson, Varieties for cohomology with twisted coefficients, Acta Math. Sin. (Engl. Ser.) 15 (1999), 81-92. MR 2000f:20087

[C2] J. F. Carlson, The thick subcategory generated by the trivial module, Infinite Length Modules, ed. Henning Krause and Claus Michael Ringel, Trends in Mathematics, Birkhäuser Verlag (2000), 285-296. MR 2001k:20005

[CR] J. F. Carlson and G. R. Robinson, Varieties and modules with vanishing cohomology, Math. Proc. Camb. Phil. Soc. 116 (1994), 245-251. MR 95c:20073

[CM] D. H. Collingwood and W. M. McGovern, Nilpotent Orbits in Semisimple Lie Algebras, Van Nostrand Reinhold, 1993. MR 94j:17001

[CNP] J. F. Carlson, D. K. Nakano and K. M. Peters, On the vanishing of extensions of modules over reduced enveloping algebras, Math. Annalen 302 (1995), 541-560. MR 96f:17029

[FP1] E. M. Friedlander and B. J. Parshall, Support varieties for restricted Lie algebras, Invent. Math. 86 (1986), 553-562. MR 88f:17018 
[FP2] E. M. Friedlander and B. J. Parshall, Geometry of $p$-unipotent Lie algebras, J. Algebra 109 (1987), 25-45. MR 89a:17017

[FS] E. M. Friedlander and A. A. Suslin, Cohomology of finite group schemes over a field, Invent. Math. 127 (1997), no. 2, 209-270. MR 98h:14055a

[Hum] J. E. Humphreys, Conjugacy Classes in Semisimple Algebraic Groups, American Mathematical Society (Mathematical Surveys and Monographs), 1995. MR 97i:20057

[Jan] J. C. Jantzen, Representations of algebraic groups, Academic Press, 1987. MR 89c:20001

[NPV] D. K. Nakano, B. J. Parshall and D. C. Vella, Support varieties for algebraic groups, J. Reine Angew. Math. 547 (2002), 15-49. MR 2003b:20063

[Qu] D. G. Quillen, The spectrum of an equivariant cohomology ring I, II, Ann. of Math. 94 (1971), 549-572, 573-602. MR 45:7743

[SFB1] A. A. Suslin, E. M. Friedlander and C. P. Bendel, Infinitesimal one-parameter subgroups and cohomology, Jour. AMS. 10 (1997), 693-728. MR 98h:14055b

[SFB2] A. A. Suslin, E. M. Friedlander and C. P. Bendel, Support varieties for infinitesimal group schemes, Jour. AMS. 10 (1997), 729-759. MR 98h:14055c

Department of Mathematics, University of Georgia, Athens, Georgia 30602

E-mail address: djb@byrd.math.uga.edu

Department of Mathematics, University of Georgia, Athens, Georgia 30602

E-mail address: nakano@math.uga.edu 